\title{
Performance Evaluation of two Port and four Port Measurement for Twisted Pair Cable
}

\author{
Azhari Bin Asrokin', Mohamad Kamal Bin A. Rahim² \\ ${ }^{1}$ Telekom Research \& Development Sdn Bhd, 63000 Cyberjaya Selangor, Malaysia \\ ${ }^{2}$ Advanced RF and Microwave Research Group, Faculty of Electrical Engineering, \\ Universiti Teknologi Malaysia, 81310 UTMJB Johor, Malaysia
}

\begin{tabular}{l} 
Article Info \\
\hline Article history: \\
Received Apr 8, 2018 \\
Revised Jul 20, 2018 \\
Accepted Jul 27, 2018 \\
\hline
\end{tabular}

\section{Keyword:}

Balun transformer

Differential signal

Network analyzer

Scattering parameters

Twisted Pair Cable

\begin{abstract}
A balance-unbalance (balun) transformer is commonly used to connect the balance $100 \mathrm{Ohm}$ twisted pair cable to the unbalance $50 \mathrm{Ohm}$ network analyzer ports, but due to the limitations of the core (i.e. ferrite) inside the balun, the balun can only effectively operates at a certain band of frequencies. This limitation can be eliminated by using a 4-port vector network analyzer (VNA) which is done by connecting the VNA's ports to each conductor end. The extracted S-parameters will then be transformed to a 2-port S-parameters in differential mode at both ports. To validate the measurement technique, S-parameter measurement by using the 4-Port Network Analyzer without any balun will be compared to the measurement which used the 2-Port Network Analyzer with the balun transformers. Two twisted pair cable distances are selected as reference which are 500, and 1000 meters with nominal copper diameter of $0.5 \mathrm{~mm}$. Based on the measurement results, the 4-ports measurement shows good correlation with the 2-ports measurement especially at $500 \mathrm{~m}$ distance. This shows that the 4-ports measurement setup is suitable to be used to measure twisted pair copper cable and possible to measure at a higher frequency band such as up to 500 $\mathrm{MHz}$ but at a shorter twisted pair cable distance.
\end{abstract}

Copyright $@ 2018$ Institute of Advanced Engineering and Science. All rights reserved.

\section{Corresponding Author:}

Azhari Bin Asrokin,

Communication Technologies Division,

Telekom Research \& Development Sdn Bhd,

Lingkaran Teknokrat Timur, 63000 Cyberjaya, Selangor, Malaysia.

Email: azhari.asrokin@tmrnd.com.my

\section{INTRODUCTION}

Twisted pair cable was invented and patented by Alexander Graham Bell in the 1880s [1] to transmit voice over the wire. Since then, the function of twisted pair copper has changed from voice to facsimile and in the last decade the twisted pair is widely used to transmit digital data alongside with fibre cable. Eventhough a study by [2] stated that twisted pair may have less effect on reducing external noise, the claim can only be true for short distances only, but for long distances, the effect of reducing external noise is greatly noticeable. Twisted pair cable was thought going to end with the ISDN technology which can only carry data rate speed below $1 \mathrm{Mbps}$, but then Asymetric Digital Subscriber Line, ADSL was introduced in the early 1990s and was then standardize in 1999 in ITU-T [3]. Later, the DSL family expanded with HighSpeed Digital Subscriber Line, HDSL, Symetrical High-Speed Digital Subscriber Line, SHDSL, Very HighSpeed Digital Subscriber Line, VDSL, VDSL2 [4] and the latest addition, Gigabit Fast Access to Service Terminals, G.fast [5] which after several trials have shown potential of delivering aggregated data rate up to 1 Gbps [6]. As stated by [7], with current advancement of copper technology, it is difficult to phase out copper entirely from the service provider network. The capability of high data rate being offered by the mentioned xDSL technologies is only limited to the length of the twisted pair cable, such as ADSL2+ can 
offer up to $20 \mathrm{Mbps}$ with coverage distance of $5 \mathrm{~km}$, VDSL2 can offer up to 50Mbps with coverage distance of $2 \mathrm{kmand}$ G.fast can offer up to $1 \mathrm{Gbps}$ aggregated data rate with coverage distance of $250 \mathrm{~m}$ [8]. The tradeoff between data rate and coverage distance is an unavoidable limitation which commonly faced in wireless technology environment.

One way to estimate the capacity of any transmission medium is by measuring the scattering parameters (S-parameters) of the medium, take twisted pair cable for example. The S-parameter is able to describe the electrical properties of a device with any number of ports opening by observing the power coming in and out from the ports available in the device [9]. The S-parameter can be used to extract the primary parameters of the device such as twisted pair cable to observe the RLGC values of the cable [10]. An example of how the S-parameters is achieved in a two-port device is as shown in Figure 1 where S11 is obtained by observing the ratio of power coming out to port A to the power coming in from port A. While $\mathrm{S} 21$ is achieved by observing the ratio of power coming out to port B to the power coming in from port A. S21 is closely related to the channel capacity of the medium and the S-parameter is usually defined in decibel, dB unit. Equation (1) summarizes the S-parameter equation in relation to the power in and out of the ports. For a four-port device as shown in Figure 2, the S-parameter will be more complex as it will generate a four-by-four matrix as shown in Equation (2).

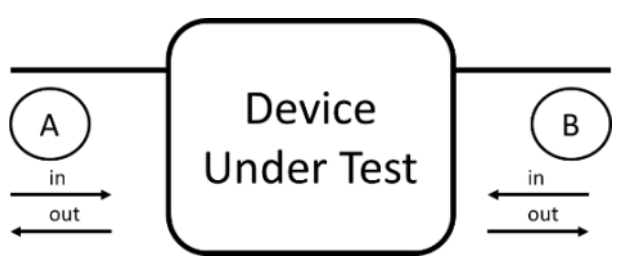

Figure 1. S-parameter for two ports

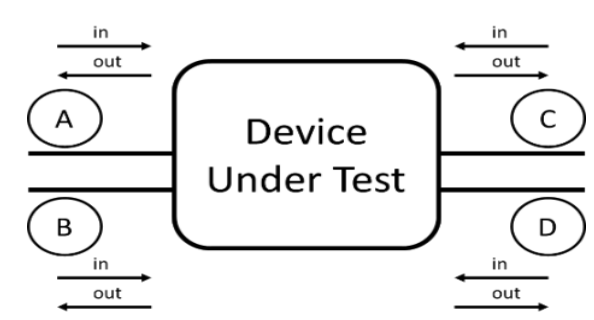

Figure 2. S-parameter for four ports

$$
\begin{aligned}
& S_{11}=\frac{A_{\text {out }}}{A_{\text {in }}} \quad S_{12}=\frac{A_{\text {out }}}{B_{\text {in }}} \\
& S_{21}=\frac{B_{\text {out }}}{A_{\text {in }}} \quad S_{22}=\frac{B_{\text {out }}}{B_{\text {in }}} \\
& S_{11}=\frac{A_{\text {out }}}{A_{\text {in }}} \quad S_{12}=\frac{A_{\text {out }}}{B_{\text {in }}} \quad S_{13}=\frac{A_{\text {out }}}{C_{\text {in }}} \quad S_{14}=\frac{A_{\text {out }}}{D_{\text {in }}} \\
& S_{21}=\frac{B_{\text {out }}}{A_{\text {in }}} \quad S_{22}=\frac{B_{\text {out }}}{B_{\text {in }}} \quad S_{23}=\frac{B_{\text {out }}}{C_{\text {in }}} \quad S_{24}=\frac{B_{\text {out }}}{D_{\text {in }}} \\
& S_{31}=\frac{C_{\text {out }}}{A_{\text {in }}} \quad S_{32}=\frac{C_{\text {out }}}{B_{\text {in }}} \quad S_{33}=\frac{C_{\text {out }}}{C_{\text {in }}} \quad S_{34}=\frac{C_{\text {out }}}{D_{\text {in }}} \\
& S_{41}=\frac{D_{\text {out }}}{A_{\text {in }}} \quad S_{42}=\frac{D_{\text {out }}}{B_{\text {in }}} \quad S_{43}=\frac{D_{\text {out }}}{C_{\text {in }}} \quad S_{44}=\frac{D_{\text {out }}}{D_{\text {in }}}
\end{aligned}
$$

This paper aims to validate the use of coaxial-to-DE9 (D-subminiature electrical connector for 9-pin) adaptor locally made in-house during the S-parameter measurement by comparing S21 results of the coaxial-to-DE9 adaptor versus on the shelf balun transformer. The comparison was made by using root mean square error, RMSE method to quantify the deviation between both setup of measurement as being used in [11].

\section{RESEARCH METHOD}

In this section, the process of calibration and measuring the S-parameters by using the two-port Network Analyzer and four-port Network Analyzer is explained. The measurement involved three stages as shown in Figure 3, which are the setup of cable and equipment, measurement execution and the postprocessing of the S-parameter results and the correlation study. 


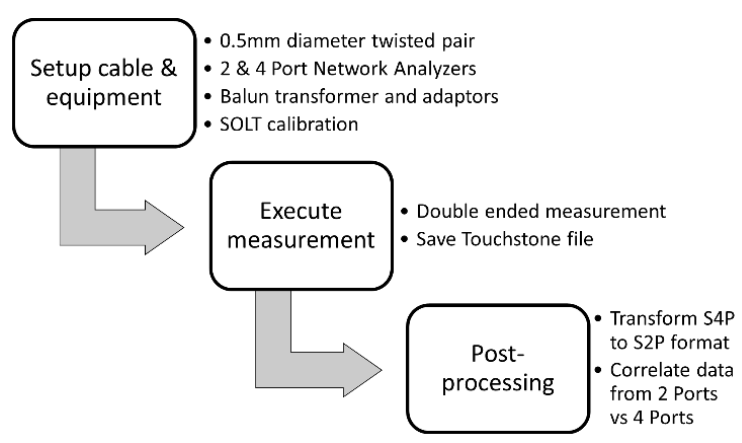

Figure 3. Overall flow of the measurement

\subsection{Cable under test}

Telekom Malaysia twisted pair cable will be used for the test with cable distance of $500 \mathrm{~m}$ and $1000 \mathrm{~m}$. The type of twisted pair cable used in the test is the overhead type with integral bearer with conductor diameter of $0.5 \mathrm{~m}$. Five different cable conditions was set to emulate the conditions which is possible to happen in the TM cable network as shown in Table 1.

Table 1. Cable Conditions Emulated for the S-Parameter Test

\begin{tabular}{|c|c|c|}
\hline $\begin{array}{c}\text { Cable } \\
\text { Condition }\end{array}$ & \multicolumn{1}{|c|}{ Cable setup } \\
\hline Ideal & Normal cable condition \\
\hline Bridge Tap & $\begin{array}{l}\text { Parallel wiring, T- } \\
\text { junction setup usually } \\
\text { occurred at the } \\
\text { customer's premise }\end{array}$ \\
\hline Open & $\begin{array}{l}\text { One of the conductor } \\
\text { of the twisted pair is } \\
\text { cut open at any part of } \\
\text { the cable }\end{array}$ \\
\hline Short & $\begin{array}{l}\text { The two conductor is } \\
\text { shorted at any part of } \\
\text { the cable }\end{array}$ \\
\hline $\begin{array}{l}\text { Uneven } \\
\text { Length }\end{array}$ & $\begin{array}{l}\text { One of the conductor } \\
\text { of the twisted pair has } \\
\text { additional cable length } \\
\text { at any part of the cable }\end{array}$ \\
\hline
\end{tabular}

Each of the cable's end was soldered to DE9 male connector as shown in Figure 4. Each end of the twisted pair conductor was soldered to pin number 5 and 9 only. Once soldered, the connectivity of each conductor was tested with digital multi-meter.

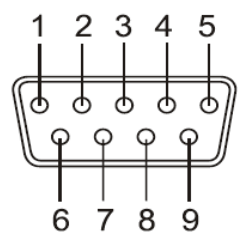

Figure 4. DE9 male connector

\subsection{Measurement setup}

The two-port measurement as shown in Figure 5 includes two balun transformers made by North Hills, model NH14211 with operating frequency from $100 \mathrm{kHz}$ to $350 \mathrm{MHz}$. The four-port measurement as 
shown in Figure 6 uses a custom made coaxial-to-DE9 adaptor which links the four coaxial cables to each of the twisted pair cable's end. The two and four port measurement was done by using Keysight (formerly known as Agilent) E5071C Network Analyzer. The measurement setting on the network analyser is as follows: start frequency at $9 \mathrm{kHz}$; stop frequency: $500 \mathrm{MHz}$ and sampling number: 10000 points.

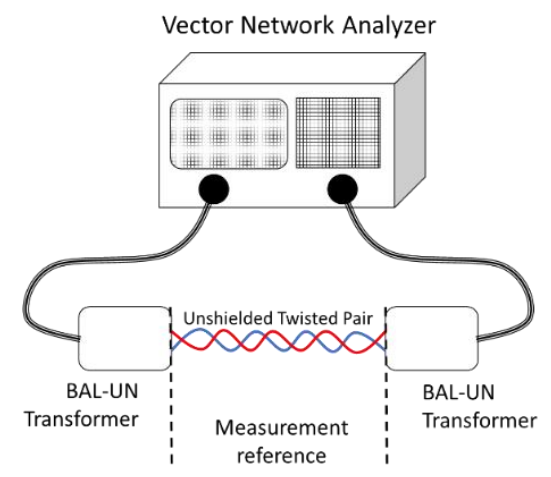

Figure 5. Two-port measurement setup

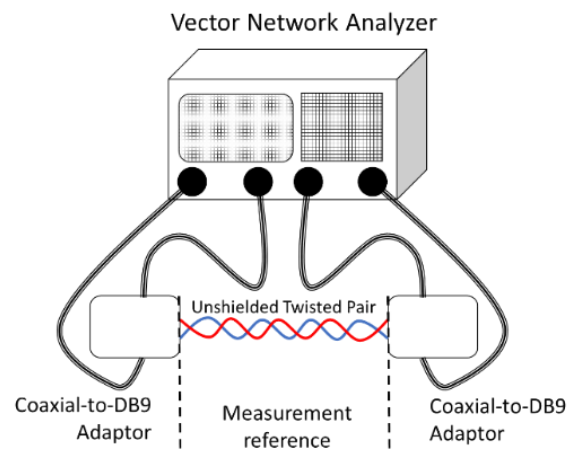

Figure 6. Four-port measurement setup

\subsection{Calibration process}

Both two-port or four-port measurement used the Short-Open-Load-Thru (SOLT) calibration method. The calibration kit was customised for DE9 connector. As shown in Figure 7, the two-port measurement, there are four calibration kit, which are the Short, Open, Load and Thru, while for the four-port measurement, the only difference from the two-port is the Thru kit will have 5 configurations as shown in Figure 8.

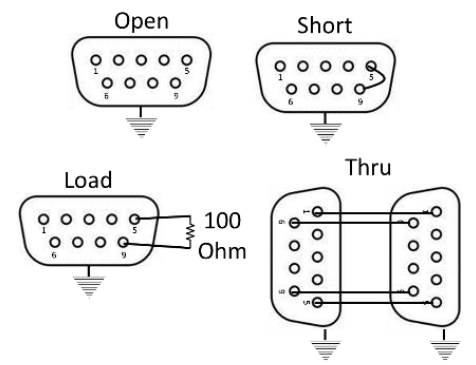

Figure 7. Calibration kit for two-port measurement
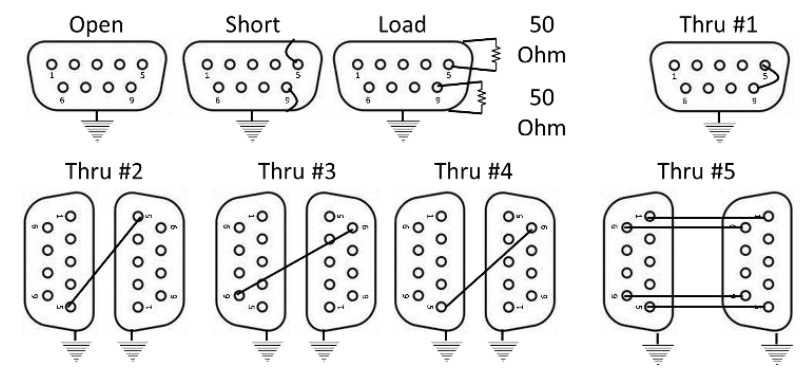

Figure 8. Calibration kit for four-port measurement

\subsection{Conversion from $S 4 P$ file to $S 2 P$ file}

The result that will be compared between the two-port and the four-port is the $\mathrm{S} 21$ parameter in $\mathrm{dB}$ unit. But before the comparison can be made, the S4P file need to be converted to S2P file. This can be done easily if the built-in function in Matlab software, S2SDD is used, which is available in the RF Toolbox. Without the use of the Matlab built-in function, the data can also be converted by following Equation (3) as mentioned in [12]. Once the S4P have been converted, the result of the S21will be compared by using root mean square method which can be calculated by using Equation (4), where A is the value of the S21.

$$
\begin{aligned}
& S_{d d 11}=\frac{S_{11}-S_{12}-S_{21}+S_{22}}{2} \\
& S_{d d 12}=\frac{S_{13}-S_{14}-S_{23}+S_{24}}{2} \\
& S_{d d 21}=\frac{S_{31}-S_{32}-S_{41}+S_{42}}{2} \\
& S_{d d 22}=\frac{S_{33}-S_{34}-S_{43}+S_{44}}{2}
\end{aligned}
$$


$R M S E=\sqrt{\frac{\sum_{f_{1}-f_{N}}\left[A_{S 4 P}-A_{S 2 P}\right]^{2}}{N}}$

\section{RESULTS AND ANALYSIS}

After the measurement for both two-port and four-port were completed, the four-port measurement will need to be converted to S2P format first. The conversion process will follow Equation (3) rule as mentioned in the previous chapter. The results and discussion chapter is presented in four parts. The first and second part is the $500 \mathrm{~m}$ and $1000 \mathrm{~m} \mathrm{~S} 21$ comparison between the two-port and four-port measurement. The third part is the root mean square error calculation tabulation between every cable conditions. Lastly, the results comparison will be discussed and the hypothesis drafted in introduction will be concluded.

\section{1. $500 \mathrm{~m}$ result comparison}

Figure 9 to Figure 13 shows the results for the five different cable conditions. For $500 \mathrm{~m}$, it is observed that most of the cable condition gives workable operating frequency (value S21 higher than $-60 \mathrm{~dB}$ ) up until $30 \mathrm{MHz}$. It is also spotted that the measurement will began to deviate more after $45 \mathrm{MHz}$ where the S2P measurement notch up a bit than the S4P measurement.

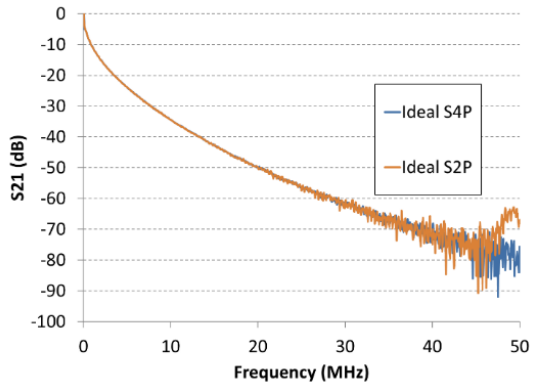

Figure 9. Result comparison for $500 \mathrm{~m}$ ideal cable condition

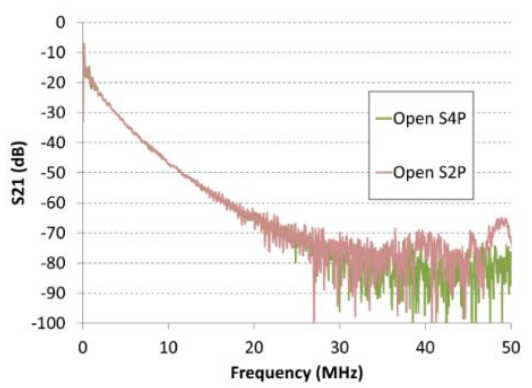

Figure 11. Result comparison for 500m Open cable condition

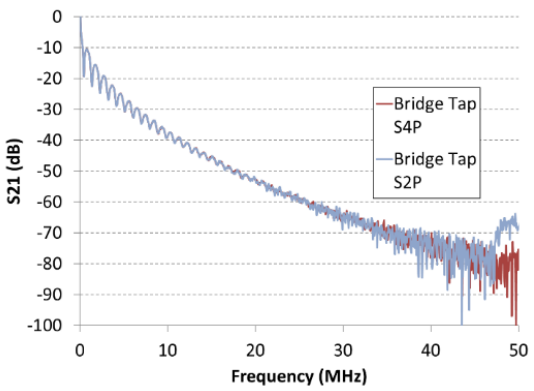

Figure 10. Result comparison for 500m Bridge Tap cable condition

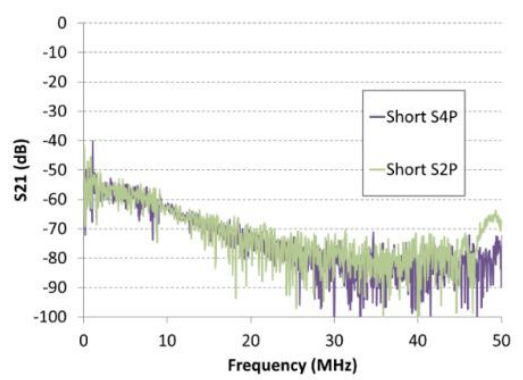

Figure 12. Result comparison for 500m Short cable condition

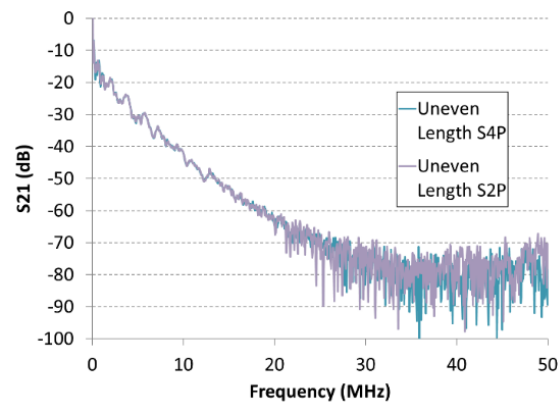

Figure 13. Result comparison for 500m Uneven Length cable condition 


\section{2. $1000 \mathrm{~m}$ result comparison}

Figure 14 to Figure 18 shows the results for $500 \mathrm{~m}$ distance, which is observed that most of the cable condition gives workable operating frequency (value S21 higher than -60dB) up until $7 \mathrm{MHz}$. The S21 values for $1000 \mathrm{~m}$ are as expectedly much lower than the $500 \mathrm{~m} \mathrm{~S} 21$ value. The ripple effect on the bridge tap condition can still be observed at $1000 \mathrm{~m}$.

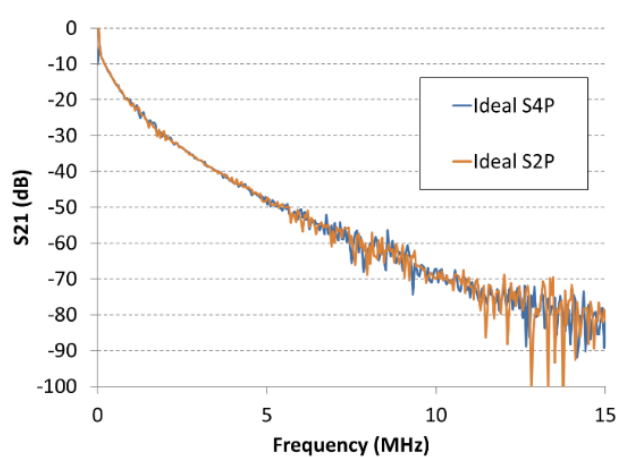

Figure 14. Result comparison for $1000 \mathrm{~m}$ ideal cable condition

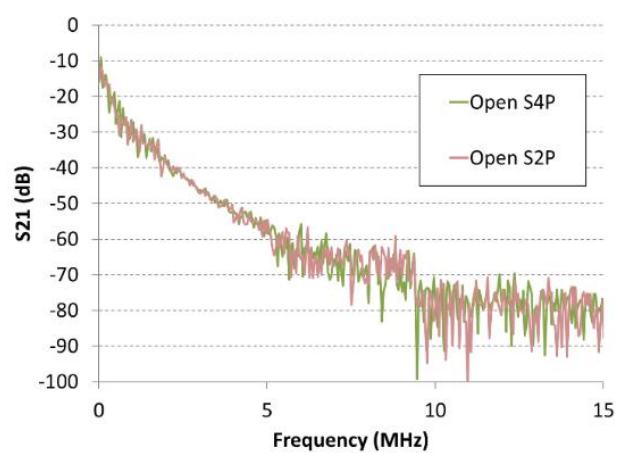

Figure 16. Result comparison for $1000 \mathrm{~m}$ open cable condition

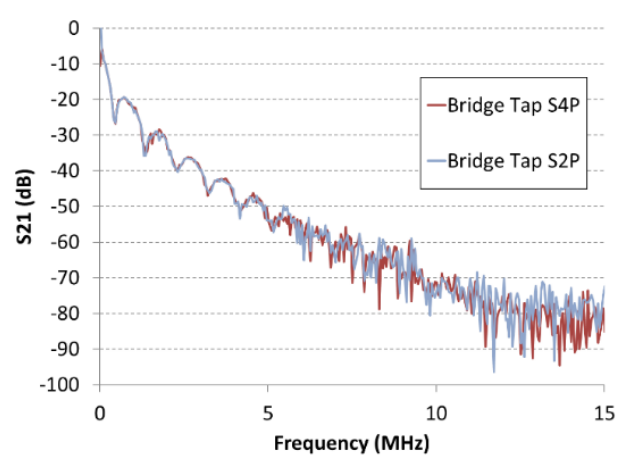

Figure 15. Result comparison for $1000 \mathrm{~m}$ bridge tap cable condition

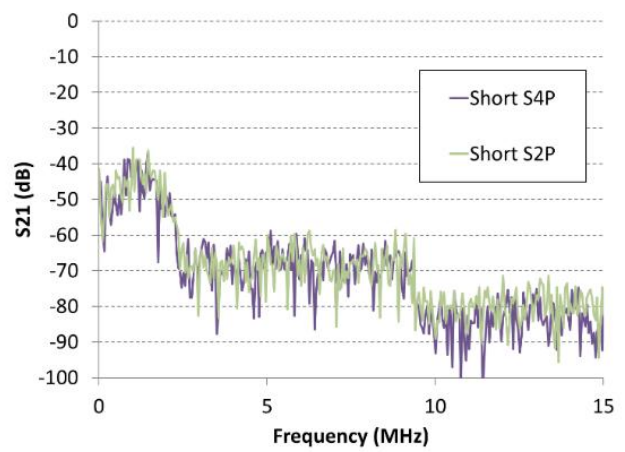

Figure 17. Result comparison for $1000 \mathrm{~m}$ short cable condition

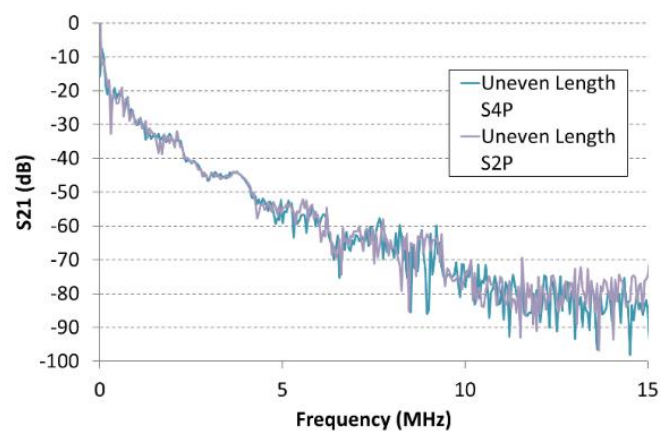

Figure 18. Result comparison for 1000m uneven length cable condition

\subsection{Root mean square error calculation and comparison}

By using Equation (4), the RMSE for each cable condition was calculated, tabulated and shown in Figure 19 and Figure 20 for distance of $500 \mathrm{~m}$ and $1000 \mathrm{~m}$ respectively. It is observed that the RMSE value are consistently high if the RMSE calculation was made by considering the whole frequency band (up to $500 \mathrm{MHz}$ ). The RMSE value also decreases along with reduction of maximum operating frequency from $500 \mathrm{MHz}$ to $50 \mathrm{MHz}$ (for $500 \mathrm{~m}$ distance) or $15 \mathrm{MHz}$ (for $1000 \mathrm{~m}$ distance) and lastly to $5 \mathrm{MHz}$. The RMSE value for Short condition is consistently high at both distance as the overall value of S21 deteriorate much faster than other cable condition even at maximum operating frequency of $5 \mathrm{MHz}$. 


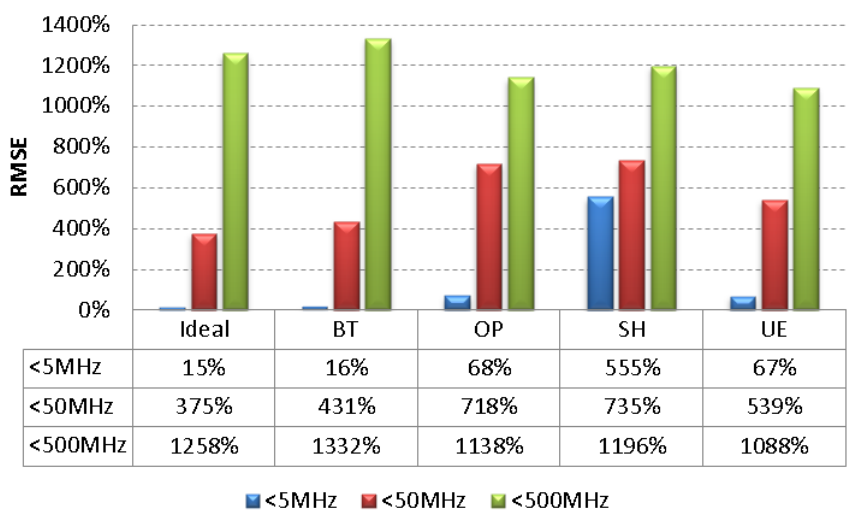

Figure 7. Root mean square error result comparison for 500m against the cable conditions

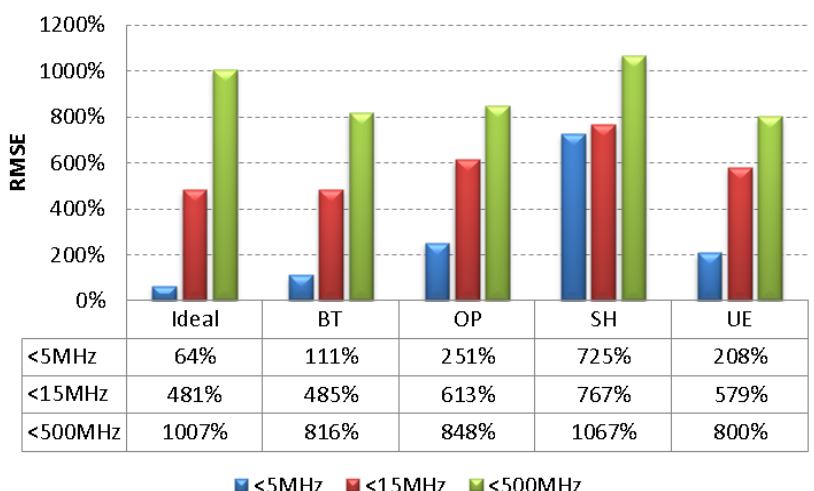

Figure 8. Root mean square error result comparison for 1000m against the cable conditions

\subsection{Discussion}

The frequency selection of $50 \mathrm{MHz}$ for $500 \mathrm{~m}$ and $15 \mathrm{MHz}$ for $1000 \mathrm{~m}$ was based on the workable frequency observed earlier in Figure 9 to Figure 18. The frequency $5 \mathrm{MHz}$ was selected to have the same reference frequency for both distance of $500 \mathrm{~m}$ and $1000 \mathrm{mso}$ that apart from having different distance, any other variables are set to be the same.

At operating frequency below $5 \mathrm{MHz}$, Ideal condition gives the best RMSE value of $15 \%$ for $500 \mathrm{~m}$ and $64 \%$ for $1000 \mathrm{~m}$ while Short condition gives the worst RMSE value of $555 \%$ for $500 \mathrm{~m}$ and $725 \%$ for $1000 \mathrm{~m}$. All S21 values for conditions other than Ideal are much lower than Ideal condition due to the impairment emulated to the twisted pair cable. Significant ripples are detected for Bridge Tap condition which is caused by the stub introduced in the somewhere in the middle of the cable.

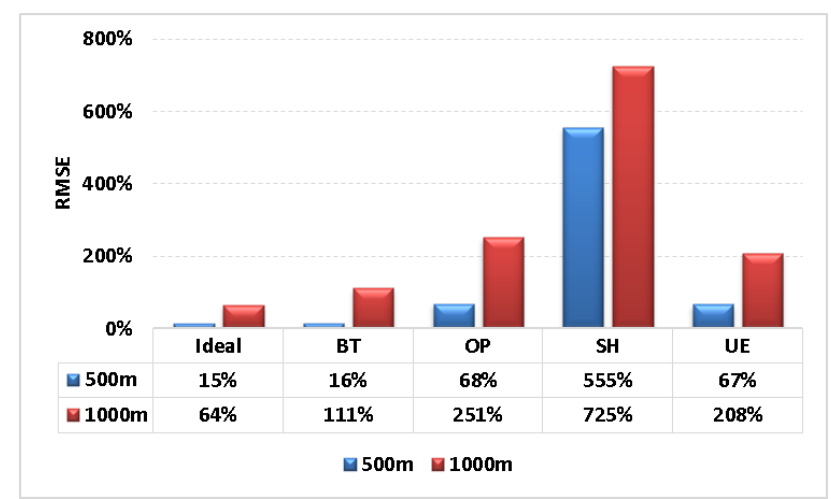

Figure 9. RMSE result comparing $\mathrm{S}_{21}$ value against the cable conditions below $5 \mathrm{MHz}$ 
It is observed that as the cable distance increase, the RMSE value will increase due to the increase of attenuation level across the frequency and thus reducing the workable operating frequency. As shown in Figure 21, even when comparing the RMSE for operating frequency below $5 \mathrm{MHz}$, the deviation at $1000 \mathrm{~m}$ distance is consistently higher than the measured 500m distance. The RMSE value below than $30 \%$ is considered good as referred to the RMSE threshold set in [13].

\section{CONCLUSION}

Based on the measurement results, the 4-ports measurement shows good correlation with the 2-ports measurement especially at $500 \mathrm{~m}$ distance. This shows that the 4-ports measurement setup is suitable to be used to measure twisted pair copper cable and possible to measure at a higher frequency band such as up to $500 \mathrm{MHz}$ but at a shorter twisted pair cable distance. This will enable the possibilities to study the potential of copper cable for higher transmission rate up to 1 Gbps data rate speed by knowing its primary and secondary property's behaviour at higher frequency bands.

\section{ACKNOWLEDGEMENTS}

The authors would like to thank Telekom Malaysia for supporting the project RDTC/170921 and special thanks to the entire team member, especially Iza Shahril, Mohd Rezadzudin, Mohd Noorfalllah, and Mr Haidil Azhar and the intern students who had given their best effort to accomplish their task within the project. The authors also thank the Ministry of Higher Education (MOHE) for supporting the research work; Research Management Centre (RMC), School of Postgraduate Studies (SPS), Communication Engineering Department, Faculty of Electrical Engineering (FKE), and Universiti Teknologi Malaysia (UTM) Johor Bahru for the support of the research work.

\section{REFERENCES}

[1] J. McBee, D. Barnett, and D. Groth, Cabling: The Complete Guide to Network Wiring. John Wiley \& Sons, 2004.

[2] N. H. Abdul Rahim, Z. Abadi Baharudin, M. N. Othman, and P. N. S. Ab Rahman, "The Comparative Study between Twisted and NonTwisted Distribution Line for Photovoltaic System Subjected to Induced Voltage Generated by Impulse Voltage," TELKOMNIKA (Telecommunication, Computing, Electronics and Control), vol. 12, no. 8, pp. 5774-5778, 2014.

[3] ITU-T, ITU-T Recommendation G.992.1: Asymmetric Digital Subscriber Line (ADSL) transceivers. Geneva, 1999.

[4] T. Starr, M. Sorbara, J. M. Cioffi, and P. J. Silverman, DSL Advances. 2002.

[5] M. Timmers, M. Guenach, C. Nuzman, and J. Maes, "G. fast: Evolving the Copper Access Network," IEEE Commun. Mag., vol. 51, no. August, pp. 74-79, 2013.

[6] S. Myers, "Gigabit Networks - The future of G.fast \& XG-FAST Services," 2016.

[7] I. Kateeb, "Should copper cables be phased out in the last mile?," Bull. Electr. Eng. Inf., vol. 1, no. 4, 2012.

[8] J. Maes and C. J. Nuzman, "The Past, Present and Future of Copper Access," Bell Labs Tech. J., vol. 20, pp. 1-10, 2015.

[9] D. M. Pozar, Microwave Engineering, 2nd Editio. John Wiley \& Sons, 1998.

[10] M. K. Sampath, "On Addressing the Practical Issues in the Extraction of RLGC Parameters for Lossy Multiconductor Transmission Lines using S-parameter Models," in 2008 IEEE-EPEP Electrical Performance of Electronic Packaging, 2008, no. 1, pp. 259-262.

[11] R. Asyrofa and W. F. Mahmudy, "Regression Modelling for Precipitation Prediction Using Genetic Algorithms," TELKOMNIKA (Telecommunication, Compututing, Electronics and Control), vol. 15, no. 3, 2017.

[12] W. Fan, A. Lu, L. L. Wai, and B. K. Lok, "Mixed-mode S-parameter characterization of differential structures," Proc. 5th Electron. Packag. Technol. Conf. EPTC 2003, pp. 533-537, 2003.

[13] R. Veerasamy, H. Rajak, A. Jain, S. Sivadasan, C. P. Varghese, and R. K. Agrawal, "Validation of QSAR Models Strategies and Importance," Int. J. Drug Des. Disocovery, vol. 2, no. 3, pp. 511-519, 2011.

\section{BIOGRAPHIES OF AUTHORS}

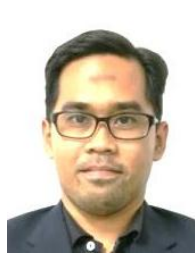

Azhari Bin Asrokin is a researcher in Telekom Research \& Development Sdn Bhd (TM R\&D) currently involves in copper technology research group. He completed his Bachelor and Master degree in Electrical Engineering (Telecommunication) from Universiti Teknologi Malaysia in 2004 and 2007 respectively. Previously, he designed antennas and RF passive devices specifically for base station antennas until 2013. In 2013 onwards, he actively involves in xDSL technologies research to support Telekom Malaysia's business specifically the copper access technologies. His work comprises of twisted pair copper cable characterization and xDSL channel modelling for next generation xDSL systems such as G.fast and Super Vector technologies. 


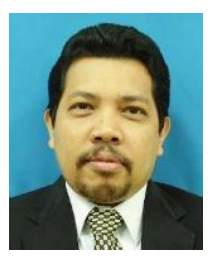

Mohamad Kamal A Rahim received the B Eng. degree in Electrical and Electronic Engineering from University of Strathclyde, UK, in 1987. In 1989, he joined the Department of Communication Engineering, Faculty of Electrical Engineering Universiti Teknologi Malaysia Kuala Lumpur as an Assistant Lecturer A. He obtained his M.Eng Science from University of New South Wales Australia in 1992 and PhD degrees in Electrical Engineering from University of Birmingham UK in 2003. After he received his Master he was appointed as a Lecturer at Faculty of Electrical Engineering. Now he is the Professor in RF and Antenna at Faculty of Electrical Engineering Universiti Teknologi Malaysia. Some of his research interest includes the areas of design of dielectric resonator antennas, microstrip antennas, RFID antennas for readers and tags, microwave circuits, EBG, artificial magnetic conductors, metamaterials, phased array antennas, computer aided design for antennas, and design of millimeter wave antennas. He has published over 300 articles in journals and conference papers. 